III Simpósio Paranaense de Modelagem.

Simulaçãoe Controle de Processos

ISSN : 1984-7521

\title{
MODELAGEM DE UM REATOR CSTR NÃO-ISOTÉRMICO PARA PRODUÇÃO DE ACETATO DE ETILA
}

\author{
Isabella J Pergher ${ }^{1}$, Isabelle Moreira ${ }^{1}$, Robson Schuarça ${ }^{1}$, Emerson Martim ${ }^{1 *}$ \\ 1 - Pontifica Universidade Católica do Paraná-PUCPR, emerson.martim@pucpr.br
}

\begin{abstract}
Resumo - No presente estudo, foi desenvolvida a modelagem de um reator CSTR não-isotérmico para a produção de acetato de etila. Foi realizado o balanço de massa e de energia em regime transiente, além dos estudos termodinâmico e cinético para essa reação. Os sistemas de equações diferenciais ordinárias, obtidas a partir dos balanços, foram solucionadas pelo método numérico de Euler. Com esses estudos, foi possível observar o comportamento da reação no reator avaliado, analisando-se as concentrações de todos os componentes e a temperatura em relação ao tempo.
\end{abstract}

Palavras-chave: acetato de etila, CSTR não-isotérmico, estudo termodinâmico, estudo cinético.

\section{1 - Introdução}

Tradicionalmente os processos eram operados de forma artesanal e empírica, contudo, com vista da necessidade do desenvolvimento de processos mais seguros, econômicos, ambientalmente viáveis e eficientes, estudos vêm sendo feitos para uma melhor compreensão dos fenômenos que ocorriam nos equipamentos e seus cálculos [1].

Tais estudos, aliados à modelagem matemática e à simulação do processo, é possível obter uma solução ótima para as condições de operação e dimensionamento do equipamento [1]. Os reatores químicos se mostram como um dos mais importantes equipamentos a serem otimizados em um processo industrial, dada a sua inerente função de obtenção dos produtos e a sua capacidade de interferir em todo o processo.

O reator contínuo de mistura perfeita - CSTR -, também conhecido como tanque agitado, é frequentemente utilizado nas indústrias químicas, possuindo a característica de proporcionar a homogeneidade das propriedades do sistema reacional em todo o seu volume e em seu efluente. Apesar de o CSTR possuir características uniformes, não é possível afirmar, entretanto, que a reação em seu interior será isotérmica, pois para o processo ser assim considerado, a temperatura de alimentação deve ser igual à do interior do reator [2].

O reator contínuo de mistura perfeita não-isotérmico (Fig. 1) é objeto de estudo desse trabalho e, para melhor representar esse comportamento, optou-se por trabalhar com a produção do Acetato de Etila, a fim de aplicar a modelagem em um caso real.

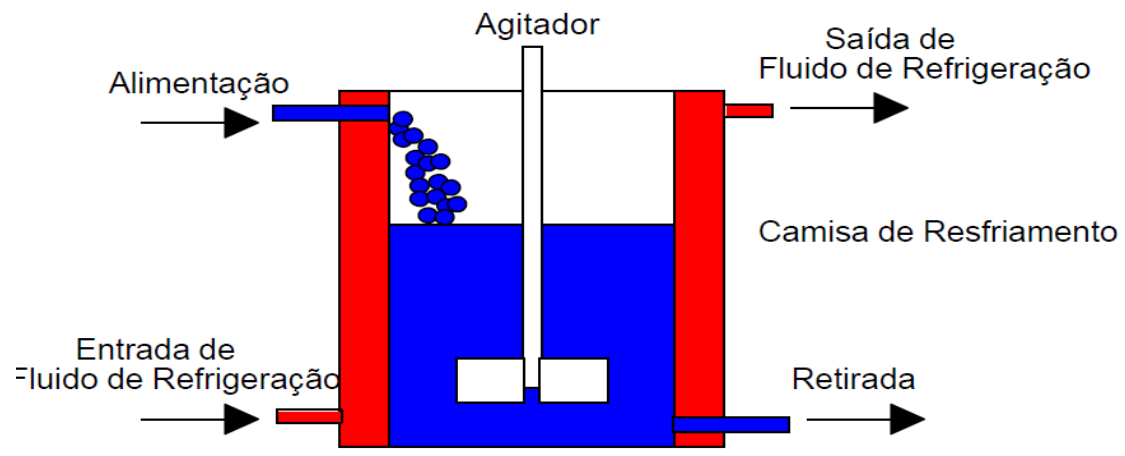

Figura 1- Reator CSTR 
O acetato de etila (ou etanoato de etila) é um composto orgânico pertencente à família dos ésteres. É muito utilizado como solvente no processo de extração do ramo alimentício, no setor industrial para produção de tintas, vernizes, filme celulósico, papel, folha de alumínio, PVC, poliéster, polietileno e polipropileno, pois possui alto poder de solvência [3].

Segundo a Patente US6693213 de 11/10/2000, um dos processos mais comuns para a produção do acetato de etila é a reação entre ácido acético e etanol, na presença de um catalisador ácido [4]. A reação citada, contida na Fig. 2, representa o processo sobre o qual será realizado o estudo cinético, termodinâmico e a modelagem [5].

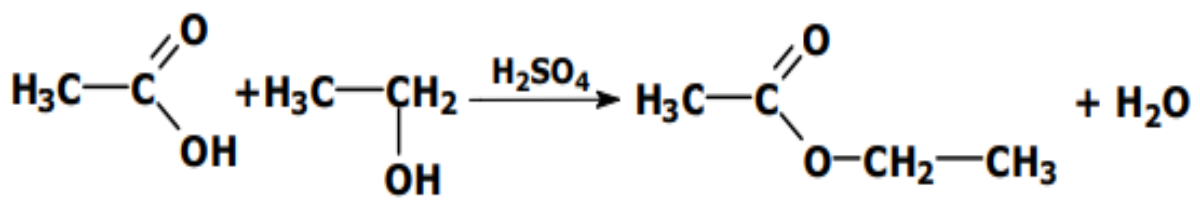

Figura 2 - Reação de obtenção de acetato de etila Fonte: Barcza, 2012.

Esterificações são facilitadas pelo aumento da temperatura do meio reacional e pela presença de um forte catalisador. A velocidade de formação do éster depende do ácido orgânico e do álcool utilizado [5].

\section{2 - Materiais e Métodos}

\subsection{Modelagem}

Para melhor entendimento e, afim de seguir os padrões de modelagem apresentados pela literatura de Fogler [2], reescreve-se a reação escolhida para o estudo (Fig. 2) de forma genérica, em função do reagente limitante (ácido acético):

$$
\mathrm{A}+\mathrm{B} \rightarrow \mathrm{C}+\mathrm{D}
$$

\subsubsection{Balanço de Massa Global}

Por meio do balanço mássico global, no qual foi admitido que o reator CSTR é bem misturado e que o fluido é incompressível, pode-se afirmar que a massa específica é a mesma em todos os pontos no interior do equipamento, inclusive na corrente de saída, sendo constante no processo. Além disso, por possuir um bom controle de nível, a variação de volume em relação ao tempo é igual a zero. Assim, prova-se que a vazão molar de entrada é igual a vazão molar de saída (Eq. 1).

$F_{o}=F$

\subsubsection{Balanço de Massa por Componente}

- Balanço de Massa para o componente A

Segue-se a lei de velocidade de reação de $2^{\mathrm{a}}$ ordem e considera-se a reação química elementar (Eq. 2), em que k é a taxa de velocidade e $C_{A}$ e $C_{B}$ são as concentrações molares dos reagentes.

$$
-r_{A}=k C_{A} C_{B}
$$


Realizando o balanço para o componente, tem-se a Eq. 3.

$\frac{d n_{A}}{d t}=F_{o} C_{A 0}-F C_{A}+R_{A} V$

Como visto no balanço global, as vazões são iguais, o volume é constante, e as concentrações no interior do reator e na corrente de saída são iguais. Sabe-se também que a quantidade molar $(n)$ pode ser escrita pela multiplicação da concentração de certo componente pelo volume, podendo-se reescrever a equação conforme Eq. 4.

$\frac{d C_{A}}{d t}=\frac{F}{V}\left(C_{A 0}-C_{A}\right)-k C_{A} C_{B}$

- Balanço de Massa para o componente B

Utilizando-se o mesmo procedimento já descrito para o componente A, alterandose apenas o valor de entrada de B chega-se na Eq. 5.

$\frac{d C_{B}}{d t}=\frac{F}{V}\left(C_{B 0}-C_{B}\right)-k C_{A} C_{B}$

- Balanço de Massa para os componentes C e D (Eq. 6-8)

$R_{C}=R_{D}=k C_{A} C_{B}$

$\frac{d C_{C}}{d t}=\frac{F}{V}\left(C_{C 0}-C_{C}\right)+k C_{A} C_{B}$

$\frac{d C_{D}}{d t}=\frac{F}{V}\left(C_{D 0}-C_{D}\right)+k C_{A} C_{B}$

\subsubsection{Balanço de Energia}

A Eq. 9 é escrita de acordo com os seguintes termos:

Energia acumulada Total $=$ Energia que entra - Energia que sai + Energia Produzida

$\frac{d E_{T}}{d t}=F_{o} \rho_{o} e_{T e}-F e_{T s} \rho+\dot{Q}+\dot{W}+\dot{R_{T}} V$

Em que $E_{T}$ é o conteúdo de energia térmica no reator em um determinado instante $t, e_{T e}$ é a quantidade energética por unidade de massa na alimentação, $e e_{T s}$ é o conteúdo energético da corrente de saída, $Q$ é a taxa de calor fornecida ao sistema (camisa) $(\mathrm{J} / \mathrm{s})$, $W$ é a taxa de trabalho executado sobre a massa de fluido no interior do reator $(\mathrm{J} / \mathrm{s})$ e $R$ é a taxa de liberação de energia térmica no sistema $\left(\mathrm{J} / \mathrm{m}^{3} \mathrm{~s}\right)$, devido a transformações químicas.

Sabe-se que $E_{T}=\rho V e_{T}, F_{0} \rho_{0} e_{T e}=F \rho e_{T e}$ e $F \rho e_{T s}=F \rho e_{T}$. Além disso, pela definição da termodinâmica, a energia de um material é proporcional à diferença das temperaturas existentes entre o estado do material e o estado de referência, possuindo coeficiente de proporcionalidade (cp - capacidade calorífica ou calor específico) (Eq. 10).

$e_{T}=c_{p}\left(T-T^{R}\right)$

Pela reação escolhida, sabe-se que a capacidade calorifica é praticamente constante e independente da composição e da temperatura do meio reacional, sendo possível reescrever a Eq. 9 conforme a Eq. 11. 
$\rho V C_{p} \frac{d T}{d t}=F \rho C_{p}\left(T_{e}-T\right)+\dot{Q}+\dot{W}+\dot{R_{T}} V$

É conhecido, ainda, que a quantidade de calor trocado entre um meio sólido e um meio fluido é proporcional à diferença entre a temperatura existente entre eles e também à área de transferência térmica (Eq. 12). O coeficiente de proporcionalidade (U) é chamado de coeficiente global de transferência de calor e Tc é a temperatura da camisa.

$Q=U A\left(T_{c}-T\right)$

O trabalho mecânico pode ser considerado desprezível quando comparado aos demais termos do balanço. A energia química é transformada em energia térmica de forma proporcional à velocidade de reação, sendo o coeficiente de proporcionalidade o calor de reação $\left(\Delta H_{R}\right)$. Substituindo-se a equação de Arrhenius (Eq. 13) na Eq. 11, obtémse a Eq. 14.

$$
\begin{aligned}
& K=K_{0} \exp ^{\left(\frac{-\Delta E}{R T}\right)} \\
& \rho V C_{p} \frac{d T}{d t}=F \rho C_{p}\left(T_{e}-T\right)+U A\left(T_{c}-T\right)+K_{0} \exp ^{\left(\frac{-\Delta E}{R T}\right)} C_{A} C_{B}\left(\Delta H_{R}\right) V
\end{aligned}
$$

\subsection{Estudo Cinético}

Para a realização do estudo cinético, separaram-se os componentes nas quantidades de 1,5 L de álcool etílico absoluto, $120 \mathrm{~mL}$ de ácido sulfúrico PA e 1,0 L de ácido acético glacial. Adicionou-se ao reator o álcool etílico e o ácido sulfúrico. Aqueceuse a mistura até $60^{\circ} \mathrm{C}$ e, posteriormente, adicionou-se lentamente o ácido acético, controlando-se a temperatura (entre 70 e $80^{\circ} \mathrm{C}$ ) e o tempo. Foram coletadas alíquotas da mistura reacional a cada 3 minutos, separando-se $30 \mathrm{~mL}$ de cada uma em erlenmeyers. A estas foi adicionada fenolftaleína, realizando-se, então, a titulação com solução de hidróxido de sódio $2 \mathrm{M}$, anotando-se cada volume gasto.

\subsection{Estudo Termodinâmico}

Foi realizado o estudo termodinâmico a partir de dados da literatura referentes à entalpia e energia livre de Gibbs de cada um dos componentes na temperatura padrão [6]. A constante de equilíbrio (K) foi determinada por meio da Eq. 15.

$K=e^{-\frac{\Delta G}{R T}}$

A fim de calcular a constante de equilíbrio nas condições laboratoriais, foi utilizada a Eq. 16, e para a obtenção da energia de ativação fez-se uso da Eq. 17, utilizando-se os dados do estudo de Kistiakawshy [8] para as condições padrões.

$$
\begin{aligned}
& \ln \left(\frac{K^{\circ}}{K}\right)=-\frac{\Delta H}{R} \times\left(\frac{1}{T^{\circ}}-\frac{1}{T}\right) \\
& \ln \left(\frac{k_{1}}{k_{2}}\right)=-\frac{E a}{R} \times\left(\frac{1}{T_{1}}-\frac{1}{T_{2}}\right)
\end{aligned}
$$

Finalmente, por Arrhenius (Eq. 13), encontrou-se a constante $K_{0}$, em m³/(mol.s). 


\section{3 - Resultados e Discussão}

\subsection{Estudo Cinético}

Para a determinação da ordem da reação, utilizou-se o método diferencial, fazendo-se uso das equações cinéticas (Eq. 18 - 20).

$$
\begin{gathered}
\text { Ordem 0: } \\
C_{A}=-K \cdot t+C_{A 0} \\
\text { Ordem 1: } \\
\ln \left(C_{A}\right)=-K \cdot t+\ln \left(C_{A 0}\right)
\end{gathered}
$$

Ordem 2:

$$
\frac{1}{C_{A}}=-K \cdot t+\frac{1}{C_{A 0}}
$$

Após a prática, pôde-se analisar os dados obtidos, verificando-se a ordem e a constante cinética da reação. Para tal, analisaram-se as três ordens, utilizando-se o método diferencial, que consistiu em plotar os gráficos de concentração versus tempo. Com isso, o gráfico mais linearizado ( $\mathrm{R}$ mais próximo a 1 ) representaria a ordem da reação. $\mathrm{O}$ resultado obtido é apresentado na Fig. 3.

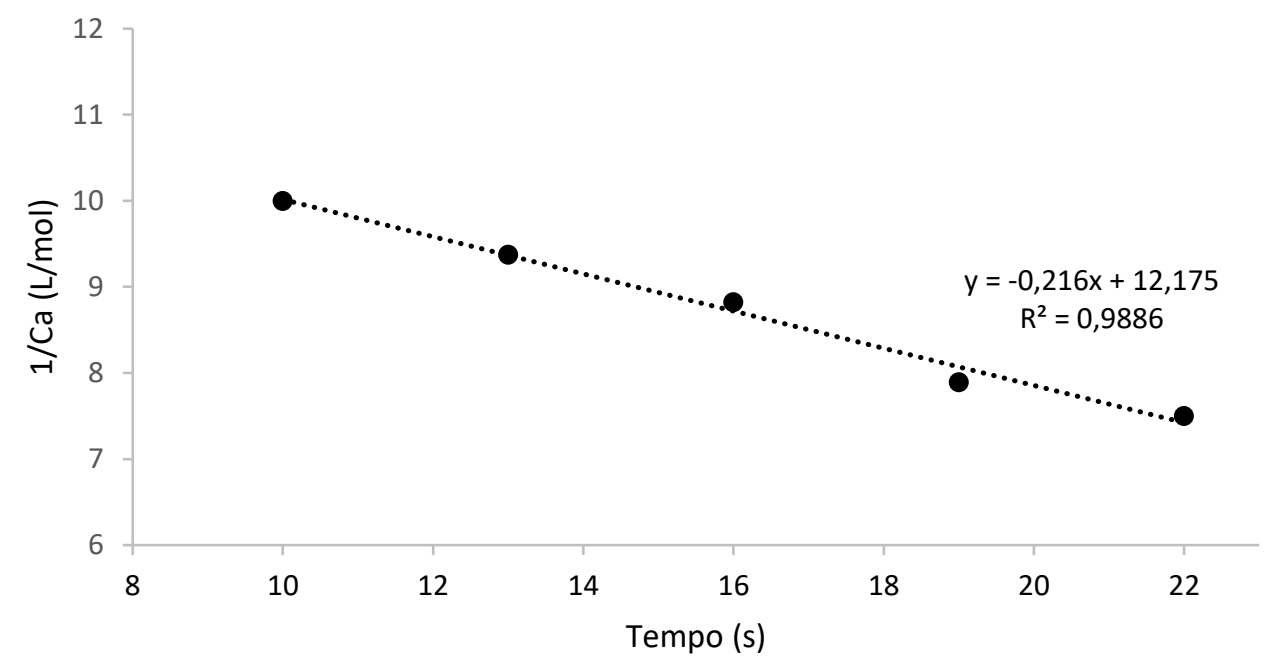

Figura 3 - Representação da $2^{\mathrm{a}}$ Ordem para a reação

Concluiu-se que a reação é de segunda ordem, podendo-se determinar a constante cinética por meio da relação da equação obtida pelo gráfico $(\mathrm{y}=-0,216 \mathrm{x}+12,175)$ com a Eq. 20. Com isso, obteve-se que a constante da reação a $70^{\circ} \mathrm{C}$ é igual a $0,216 \mathrm{~L} /(\mathrm{mol} . \mathrm{min})$.

A entalpia calculada foi de $-5880 \mathrm{~J} / \mathrm{mol}$, representando uma reação exotérmica, enquanto a energia livre de Gibbs foi de $-5430 \mathrm{~J} / \mathrm{mol}$, sendo espontânea. A partir desses valores, chegou-se à constante de equilíbrio nas condições padrões, sendo igual a 8,940, e na condição operacional $\left(70^{\circ} \mathrm{C}\right)$ (Eq. 16), equivalente a 6,550.

A fim de mensurar a energia de ativação, foi necessário calcular a constante de velocidade. Para tanto, utilizou-se o estudo de Kistiakawshy, verificando-se um valor de 0,001 L/(mol.min). Relacionando com o dado da constante de velocidade obtido na Fig. 3 , para a temperatura experimental, igual a 0,216 (L/mol.min), determinou-se a energia de ativação, sendo esta igual a 101.605,52 J/mol. 
Dessa forma, obteve-se a constante de Arrhenius, cujo valor é de 1,0455 $\times 10^{10}$ $\mathrm{m}^{3} /($ mol.s $)$.

Os dados de entrada da simulação, realizada no excel pelo método numérico de Euller, estão contidos na Tabela 1. Eles foram obtidos dos cálculos dos estudos cinético e termodinâmico realizados e da literatura de Perry (1987) [7].

Tabela 1 - Dados de Entrada do programa

\begin{tabular}{ccc}
\hline Entrada & Quantidade & Unidade \\
\hline Concentração Inicial de A & 1000 & $\mathrm{~mol} / \mathrm{m}^{3}$ \\
\hline Concentração Inicial de B & 1500 & $\mathrm{~mol} / \mathrm{m}^{3}$ \\
\hline Concentração Inicial de C & 0 & $\mathrm{~mol} / \mathrm{m}^{3}$ \\
\hline Concentração Inicial de D & 0 & $\mathrm{~mol} / \mathrm{m}^{3}$ \\
\hline Volume do reator & 25 & $\mathrm{~m}^{3}$ \\
\hline Área de transferência & 1 & $\mathrm{~m}^{2}$ \\
\hline Vazão no reator & 0,03 & $\mathrm{~m} / \mathrm{s}$ \\
\hline T. entrada fluido no reator & 298,15 & $\mathrm{~K}$ \\
\hline T. inicial no reator & 333,15 & $\mathrm{~m} /(\mathrm{mol} . \mathrm{s})$ \\
\hline Constante de Arrhenius & $1,0455 \mathrm{E}+10$ & $\mathrm{Jg} / \mathrm{m}^{3}$ \\
\hline Massa específica & 930,8125 & $\mathrm{~J} /\left(\mathrm{K} . \mathrm{m}^{2} . \mathrm{s}\right)$ \\
\hline Energia de Ativação & 101605,52 & $\mathrm{~K} /(\mathrm{mol} . \mathrm{K})$ \\
\hline Coef. Global de tr. térmica & 20934 & $\mathrm{~J} / \mathrm{mol}$ \\
\hline Constante Univ. dos Gases & 8,314 & $\mathrm{~K}$ \\
\hline$\Delta \boldsymbol{H} \boldsymbol{K}$ & -5880 & $\mathrm{~K}$ \\
\hline T. da água efluente da camisa & 313,15 & $\mathrm{~J} / \mathrm{K}$ \\
\hline T. entrada da água na camisa & 313,15 & $\mathrm{~J} /(\mathrm{kg} \cdot \mathrm{K})$ \\
\hline Capacitância térmica na camisa & 2093400 & $\mathrm{~kg} / \mathrm{s}$ \\
\hline Capacidade calorífica da água & 4180 & $\mathrm{~s}$ \\
\hline Vazão mássica na camisa & 3 &
\end{tabular}

Fonte: PERRY, 1987; Os Autores, 2018.

O resultado da simulação dos balanços de massa e de energia estão apresentados nas Figuras 4 e 5.

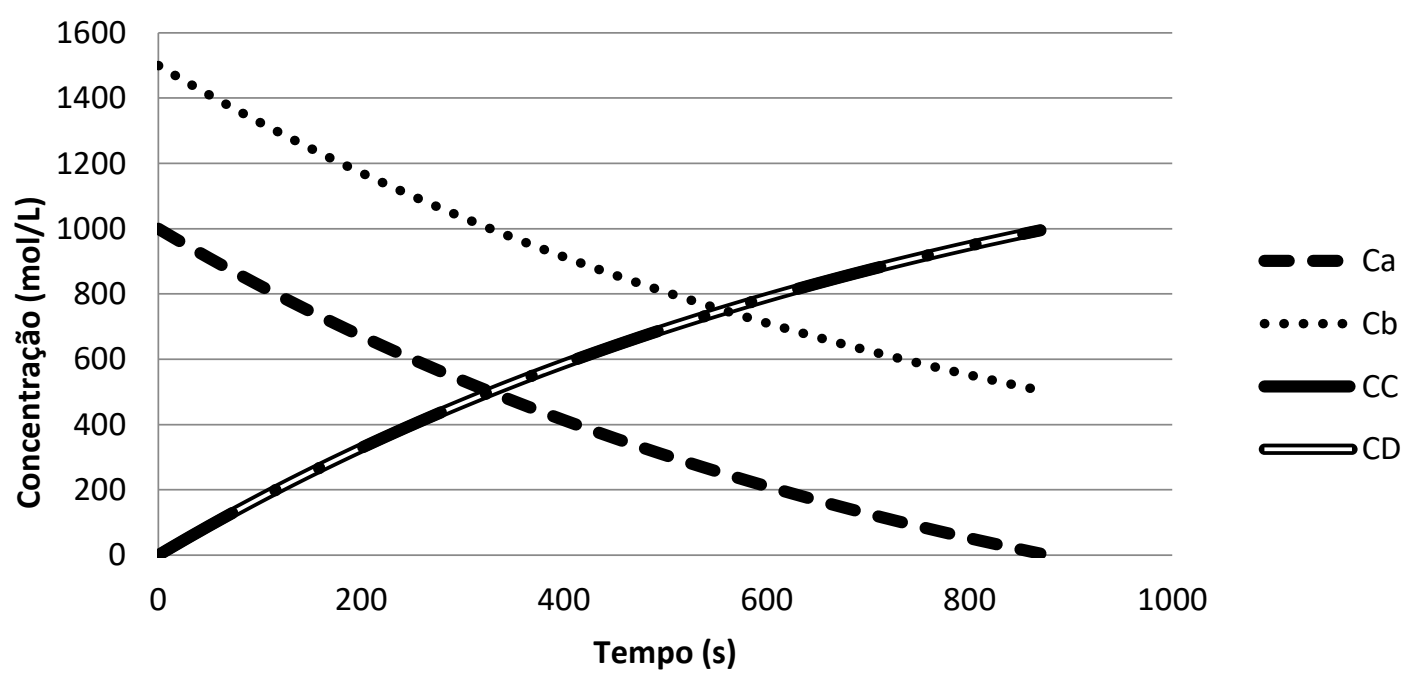

Figura 4 - Curva de concentrações de cada componente em função do tempo 


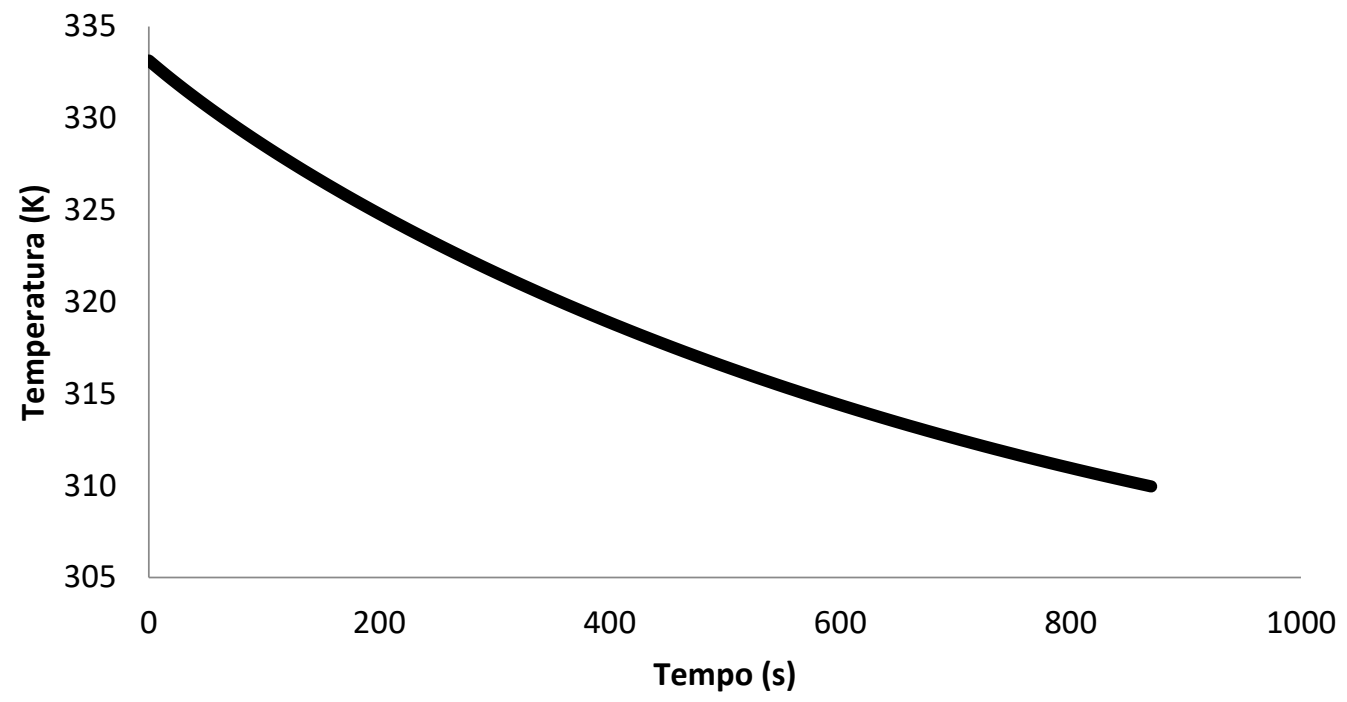

Figura 5 - Variação da temperatura no reator em função do tempo

A elementaridade foi verificada, descrita por um comportamento de $2^{\mathrm{a}}$ ordem, havendo formação equivalente de acetato de etila e água, na mesma proporção de consumo dos reagentes, conforme visualizado na Fig. 4.

A reação de formação do acetato de etila, ainda que exotérmica, não apresentou grandes variações de temperatura ao longo do tempo (Fig. 5), mesmo quando retirado o termo referente à camisa de resfriamento. Com isso, em estudos futuros, a simulação será executada para reações mais exotérmicas, a fim de se verificar uma variação maior da temperatura no decorrer da formação dos produtos, e a influência da camisa no reator.

\section{4 - Conclusão}

Por meio do estudo termodinâmico e cinético realizado, a partir de um ensaio em que houve verificação da ordem e demais parâmetros da reação escolhida, e pela modelagem e simulação do reator CSTR adiabático no excel, pelo método numérico de Euller, foi possível a avaliação do comportamento das concentrações de cada componente e da temperatura em função do tempo. Por não se tratar de uma reação altamente exotérmica, as curvas obtidas não apresentaram grande variação no decorrer do tempo, devendo, em estudos posteriores, fazer-se novamente a execução para sistemas com essa caraterística.

\section{5 - Agradecimentos}

Ao professor Emerson Martim, por todo o suporte e orientação e à PUCPR, pela oportunidade de realizar o estudo e ceder o laboratório e os materiais utilizados no ensaio prático. 


\section{6 - Referências}

1. Perlingeiro, C. A. G. Engenharia de processos, Editora Blucher, 2005.

2. Fogler, H. S. Elementos de Engenharia das Reações químicas, $3^{\text {a }}$ Edição, LTC Livros técnicos e científicos, 2002.

3. Frost, A. A.; pearson, G. R. Kinetics and Mechanism: A Study of homogeneous Chemical Reactions, 1. Ed. Nova Iorque: Editora Wiley, 1952.

4. Kolena et all, inventor; Sulzer Chemtech Ag, cessionário. Method of producing ethyl acetate and an equipment for carrying out this method. United States paten US6693213. 11 de out 2000.

5. Barcza, M. V. Esterificação. Disponível em: < www.dequi.eel.usp.com.br>. Acesso em: 22 de novembro de 2017.

6. Smith, j. M., Van ness, h. C. E Abbott, m. M., Introdução à Termodinâmica da Engenharia Química, 7a Ed., LTC - Livros Técnicos e Científicos Editora, Rio de Janeiro, 2007.

7. Perry, R; G, D. Perry’s Chemical Engineer's handbook. 7ª edição, 1997. P 200.

8. USP. Cinética Química. Disponível em: <http://sistemas.eel.usp.br/docentes/arquivos/5963230/LOQ4003/Lista8.pdf〉. Acesso em: 07 out. 2017. 